\title{
Distinct and essential morphogenic functions for wall- and lipo-teichoic acids in Bacillus subtilis
}

\section{Kathrin Schirner, Jon Marles-Wright, Richard J Lewis* and Jeff Errington*}

Centre for Bacterial Cell Biology, Institute for Cell and Molecular Biosciences, The Medical School, Newcastle University, Newcastle upon Tyne, UK

Teichoic acids (TAs) are anionic polymers that constitute a major component of the cell wall in most Gram-positive bacteria. Despite decades of study, their function has remained unclear. TAs are covalently linked either to the cell wall peptidoglycan (wall TA (WTA)) or to the membrane (lipo-TA (LTA)). We have characterized the key enzyme of LTA synthesis in Bacillus subtilis, LTA synthase (LtaS). We show that LTA is needed for divalent cation homoeostasis and that its absence has severe effects on cell morphogenesis and cell division. Inactivation of both LTA and WTA is lethal and comparison of the individual mutants suggests that they have differentiated roles in elongation (WTA) and division (LTA). B. subtilis has four ltaS paralogues and we show how their roles are partially differentiated. Two paralogues have a redundant role in LTA synthesis during sporulation and their absence gives a novel absolute block in sporulation. The crystal structure of the extracytoplasmic part of LtaS, solved at 2.4-Å resolution, reveals a phosphorylated threonine residue, which provides clues about the catalytic mechanism and identifies the active site of the enzyme.

The EMBO Journal (2009) 28, 830-842. doi:10.1038/

emboj.2009.25; Published online 19 February 2009

Subject Categories: cell \& tissue architecture; microbiology \& pathogens

Keywords: Bacillus subtilis; cell wall; lipoteichoic acid; LtaS; protein structure

\section{Introduction}

The bacterial cell wall is a crucial structure that represents the interface of the cell with the external medium. It protects the cell from damage, restrains the membrane against the large cellular turgor pressure and confers shape (Koch, 2006). It is essential in almost all organisms for cell division, and in pathogens it is recognized by both the innate and acquired immune systems (Dziarski, 2003). The wall needs to be enlarged to allow growth, while at all times maintaining its integrity (Koch, 1985; Höltje, 1998; Schaffer and Messner,

\footnotetext{
*Corresponding authors. RJ Lewis or J Errington, Centre for Bacterial Cell Biology, Institute for Cell and Molecular Biosciences, The Medical School, Newcastle University, Framlington Place, Newcastle upon Tyne NE2 4HH, UK. Tel.: + 44191222 8126; Fax: 0191222 7424;

E-mails: r.lewis@ncl.ac.uk or jeff.errington@ncl.ac.uk
}

Received: 6 November 2008; accepted: 16 January 2009; published online: 19 February 2009
2005; Stewart, 2005). Peptidoglycan (PG) is a major structure of the walls of virtually all bacteria (Koch, 2006; Vollmer et al, 2008) and consists of crosslinked glycan strands forming a complex meshwork surrounding the whole cell. Because of its essential role, PG is the major target for antibiotics such as $\beta$-lactams (e.g., penicillins and cephalosporins) and glycopeptides (e.g., vancomycin) (reviewed by Koch, 2003). The walls of Gram-positive bacteria have a second major component, teichoic acid (TA) (Archibald et al, 1961). TAs are equally abundant in walls but less well understood (reviewed by Neuhaus and Baddiley, 2003; Bhavsar and Brown, 2006). TAs are anionic polymers and come in two distinct forms depending on whether they are linked to the wall PG (wall TA, WTA) or to the head groups of membrane lipids (lipo-TA, LTA) (Neuhaus and Baddiley, 2003). A wide range of functions that have been proposed for TAs, including: antigenicity and innate immune recognition (Fedtke et al, 2004; Seo et al, 2008); pathogenicity (Morath et al, 2001; Weidenmaier et al, 2004; Fittipaldi et al, 2008); biofilm formation (Gross et al, 2001); efficient release of secreted proteins into the culture medium (Nouaille et al, 2004); maintenance of cation homoeostasis (Archibald et al, 1961; Heptinstall et al, 1970) and antibiotic resistance (Kristian et al, 2003; Kovacs et al, 2006). Many bacteria have both the WTA and LTA systems, which are synthesized by distinct biochemical routes, even when the actual repeating unit of the polymer is the same (Fischer, 1988). Such is the case for Bacillus subtilis strain 168, the subject of this study, in which both WTA and LTA consist of poly(glycerol phosphate) (poly(GroP)) but they are synthesized by separate, genetically distinct pathways (Fischer, 1988; Neuhaus and Baddiley, 2003). Both TAs can carry D-alanyl ester substitutions (reviewed by Neuhaus and Baddiley, 2003).

The WTA system has been relatively well characterized in recent years. The tagA- $F$ system encodes enzymes with identified functions for each catalytic step in the biosynthesis of poly(GroP) in the cytoplasm, and then the $\mathrm{ABC}$ transporter TagGH apparently exports the polymer for linkage to PG in the cell wall (Mauël et al, 1991; Lazarevic and Karamata, 1995; Neuhaus and Baddiley, 2003; Schertzer and Brown, 2003; Bhavsar et al, 2004; D'Elia et al, 2006a; Formstone et al, 2008). WTA was originally thought to be essential because deletions of genes affecting the later steps in the pathway are lethal (Bhavsar et al, 2004). However, recent results indicate that the apparent lethality is due to the accumulation of a toxic intermediate. Thus, when tagO, encoding the first enzyme in the pathway, is deleted and no intermediates accumulate, the cells are viable though severely compromised (D'Elia et al, 2006a,b). Interestingly, the B. subtilis mutants appear to be particularly affected in cell elongation (D'Elia et al, 2006a). They can still divide but lose the ability to maintain a rod shape and become swollen and almost round. This is consistent with a recent report from this lab that the enzymes of the WTA pathway appear to be 
associated with a cell elongation complex organized by the MreB (actin) system (Formstone et al, 2008). The MreB system forms helical filaments that run around the periphery of the cell and appear to insert wall material along helical tracts in such a way as to generate elongation of the rod while maintaining a constant cell diameter (Jones et al, 2001; Daniel and Errington, 2003; Stewart, 2005; CarballidoLópez, 2006; Den Blaauwen et al, 2008). B. subtilis and many other Gram-positive bacteria have three mreB paralogues, the others are known as $m b l$ (mre $\underline{B}$ like) and $m r e B H$ (mreB homologue). All three seem to have overlapping, partially redundant functions in cell elongation.

The LTA system and the genes that encode the biochemical pathway are poorly characterized (Perego et al, 1995; Gründling and Schneewind, 2007a). Shortly after this study was initiated, Gründling and Schneewind (2007b) reported the discovery of the key enzyme LTA synthase (LtaS), which catalyses the formation of poly(GroP) in Staphylococcus aureus. A homologue from B. subtilis was shown to possess the same activity and complement the otherwise lethal ltaS deletion. We found the ltaS gene independently in a screen for suppressors of the $\mathrm{Mg}^{2+}$-dependent growth defect of $\mathrm{mbl}$ mutants. We show that ltaS mutants are affected in divalent cation homoeostasis. The defect leads to shape malformations and to impaired septation and cell division. Therefore, WTA and LTA are largely specialized for different morphogenic systems, elongation and division, respectively. Systematic deletion of the three other ltaS paralogues of $B$. subtilis reveals that LTA is essential for spore formation, with a block at the time of polar division and/or progression beyond this step. Quadruple mutants of the B. subtilis ltaS paralogues are severely impaired but remain viable. Crucially, complete disruption of the WTA and LTA pathways is lethal, showing that TA synthesis is essential in B. subtilis. Finally, we have determined the crystal structure of the extracellular domain of LtaS and identified a catalytic threonine residue, with implications for the biochemical function of the enzyme and potential antibiotic targeting. While this study was in progress, Oku et al (2009) showed that LTA and WTA have overlapping and partially redundant roles needed for cell viability and various cell wall properties in Staphylococcus aureus.

\section{Results and discussion}

\section{Elimination of the LTA synthase YfIE suppresses the $\mathbf{M g}^{2+}$ dependency of $\mathbf{m b l}$ and mreB mutants}

Elsewhere we have shown that $m b l$ mutants are not viable at low $\mathrm{Mg}^{2+}$ concentrations and that mutations suppressing this phenotype can be readily obtained (Schirner and Errington, 2009). In a collection of transposon-induced suppressed mutants, we found three strains with insertions in the $y f l E$ gene. Wild-type $y f l E$ encodes a protein of 649 amino acids with a predicted molecular weight of $74 \mathrm{kDa}$. DNA sequencing showed that each insertion would disrupt the yflE open reading frame, after codons 41, 72 and 387, respectively. While this study was in progress, Gründling and Schneewind (2007b) showed that a closely related gene (79\% identical) in Staphylococcus aureus encodes LtaS. They also showed that the $y f l E$ gene of $B$. subtilis could complement the lethal phenotype of ltaS in S. aureus by restoring
LTA synthesis. Therefore, we subsequently refer to the $B$. subtilis yflE gene as ltaS.

It was not clear why disruption of ltaS suppressed the $\mathrm{Mg}^{2+}$ sensitivity of the $\mathrm{mbl}$ mutant. However, after more than two decades of speculation about the possible function of LTA, identification of a gene specifically required for LTA synthesis provided us with an important opportunity to investigate the physiological role of these polymers in B. subtilis. We first constructed a strain in which the ltaS gene was completely deleted and then confirmed that the ltaS $\mathrm{mbl}$ double deletion strain grows on plates at normal $\mathrm{Mg}^{2+}$ levels, unlike the $\mathrm{mbl}$ single mutant (Figure 1A). In liquid $\mathrm{PAB}$ medium with no added $\mathrm{Mg}^{2+}$ (Figure 1B; or in LB, not shown), the double mutant (circles) grew much better than the $m b l$ mutant (squares), although growth was slower than for the wild-type culture (diamonds). Deletion of ltaS also counteracted the typical swelling and extreme twisting of $\mathrm{mbl}$ mutant cells; instead, the double mutant appeared similar to the ltaS single mutant (Figure 1C) (see below).

mreB mutants and, to a much lesser degree $m r e B H$, have also been shown to be sensitive to $\mathrm{Mg}^{2+}$ levels (Formstone and Errington, 2005; Carballido-López, 2006). Combination of ltaS with an $m r e B$ disruption also led to the restoration of growth and morphology on nutrient agar (NA) plates, on which mreB mutants do not grow (Formstone and Errington, 2005), and in liquid PAB medium (data not shown). An ltaS mreBH double mutant was not detectably different from the
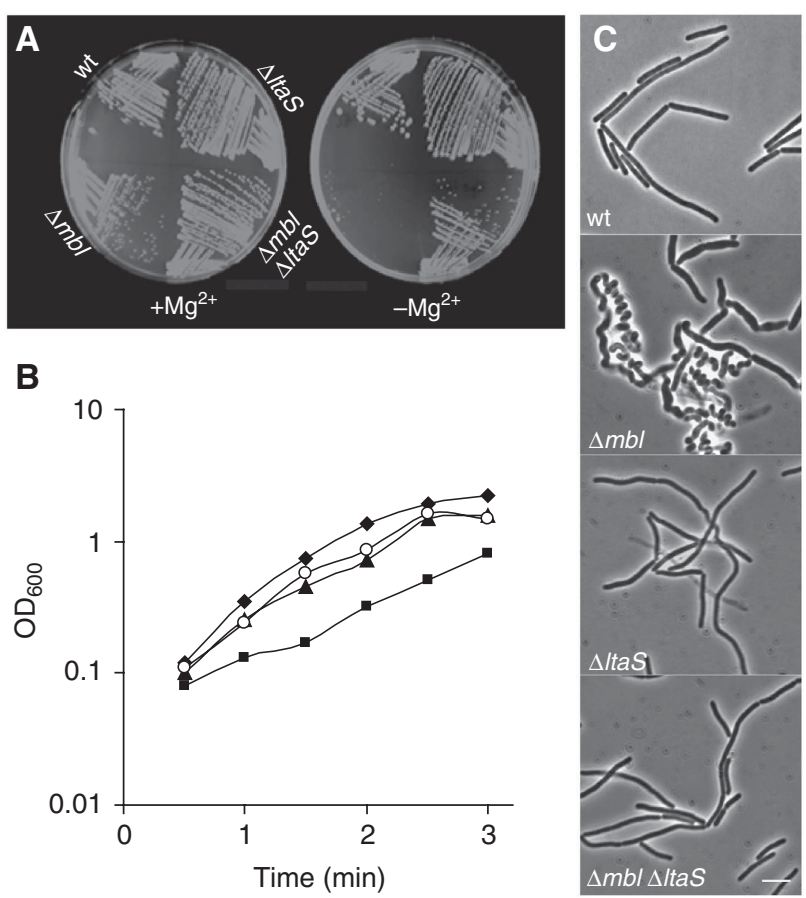

B

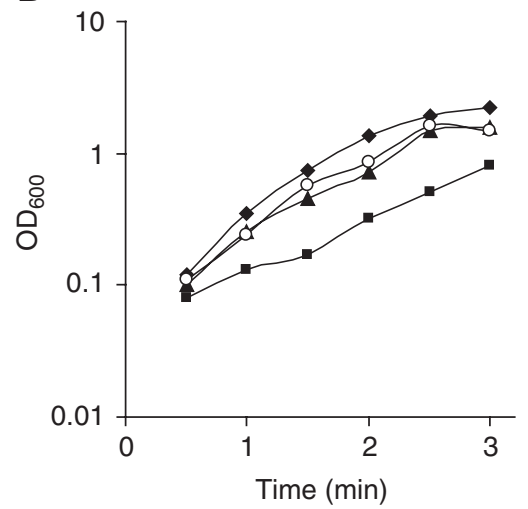
Figure 1 Deletion of ltaS suppresses the $\mathrm{Mg}^{2+}$ dependency of $\mathrm{mbl}$
mutants. (A) Growth of wild type (168), $\mathrm{mbl}$ mutant (2505), ltaS mutants. (A) Growth of wild type (168), mbl mutant (2505), ltaS
mutant (4283) and suppressed mbl mutant ( $\Delta$ mbl $\Delta l t a S, 4298)$ on NA plates with (left) or without (right) addition of $20 \mathrm{mM} \mathrm{Mg}^{2+}$. (B) Growth curves of wild type $(168, \bullet), m b l$ mutant $(2505, \mathbf{\square})$, ltaS mutant $(4283, \mathbf{\Delta})$ and suppressed $\mathrm{mbl}$ mutant $(\Delta \mathrm{mbl} \Delta \mathrm{\Delta ltaS}$, $4298, O$ ) in PAB medium at $37^{\circ} \mathrm{C}$. (C) Phase-contrast microscopy of wild type (168), mbl mutant (2505), ltaS mutant (4283) and mbl ltaS double mutant (4298) grown in PAB medium at $37^{\circ} \mathrm{C}$. Scale bar $5 \mu \mathrm{m}$. 
isogenic ltaS single mutant in growth or morphology (though the phenotype of $m r e B H$ mutants is mild).

\section{Altered susceptibility to divalent cation levels in ItaS mutants}

Although the ltaS single mutant also grew slightly more slowly than the wild type in PAB medium (doubling time 31 versus $26 \mathrm{~min}$; Figure $1 \mathrm{~B}$ ), we were surprised to find that growth was almost abolished in two of our other standard growth media, $\mathrm{CH}$ and $\mathrm{S}$-medium. By adding components of these media to $\mathrm{PAB}$, it emerged that the mutant strain was unusually sensitive to $\mathrm{Mn}^{2+}$. In the examples shown in Figure 2A, addition of $0.05 \mathrm{mM} \mathrm{MnSO}_{4}$ to NA abolished growth of the mutant, whereas growth of the wild type was

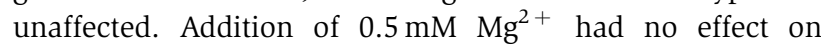
growth of the mutant, showing that the effect was not a general sensitivity to divalent cations. In fact, we noticed that the mutant had a reduced requirement for $\mathrm{Mg}^{2+}$, at least on minimal agar (Figure 2B). Thus, under these conditions the mutant strain grew better than the wild type at both 10 and $100 \mu \mathrm{M} \mathrm{Mg}^{2+}$. The lowered requirement for $\mathrm{Mg}^{2+}$ may be the reason why a deletion of ltaS suppresses the $\mathrm{Mg}^{2+}$. dependent phenotype of both $\mathrm{mbl}$ and $m r e B$ mutants (Formstone and Errington, 2005). These results provide strong support for a model in which LTA is important in scavenging and sequestration of $\mathrm{Mg}^{2+}$ ions (Neuhaus and Baddiley, 2003). Loss of the LTA-dependent buffering zone in the cell wall allows divalent cations more immediate access to the cell surface. This in turn leads to a lower requirement for $\mathrm{Mg}^{2+}$, which is a cofactor in many enzymes, and increased susceptibility to toxic $\mathrm{Mn}^{2+}$ ions, which can replace
$\mathrm{Mg}^{2+}$ because of their similar physicochemical properties but which often cannot substitute for $\mathrm{Mg}^{2+}$ in enzyme function (Cowan, 1995). These results suggest that LTA helps to provide a physicochemical environment that favours the retention of $\mathrm{Mg}^{2+}$ over $\mathrm{Mn}^{2+}$ in the cell wall.

In the process of constructing the deletion strain, we noticed that the ltaS mutant was also hypersensitive to various antibiotics (Supplementary Table 2) and lysozyme (data not shown). The effect was not specific to a certain class of antibiotic, suggesting that LTA also provides a protective layer that restricts the access of many bioactive agents to sensitive sites in the cell envelope.

\section{Defective cell division in ItaS mutants}

Microscopic examination of ltaS mutant cells grown under various conditions revealed a characteristic phenotype. This did not appear to be affected by the presence or absence of added $\mathrm{Mg}^{2+}$ or $\mathrm{Mn}^{2+}$, so all further experiments described were based on unsupplemented PAB medium unless stated otherwise. The main morphological effects (Figure 1C) were increased cell or cell chain length (B. subtilis has a tendency to form chains of cells, due to a delay between cell division and separation of sister cells; Paulton, 1971), reduced cell diameter ( $\sim 90 \%$ of that of wild-type cells; Table I) and a significant frequency of cell bending and lysis (Figure 1C). The reduced cell width has been seen previously for mutants affected in cell envelope systems (e.g., Carballido-López et al, 2006), but its significance is not yet understood. In membrane-stained preparations, it was evident that the elongated appearance was at least partly due to an effect on cell division, with long aseptate regions occurring at irregular
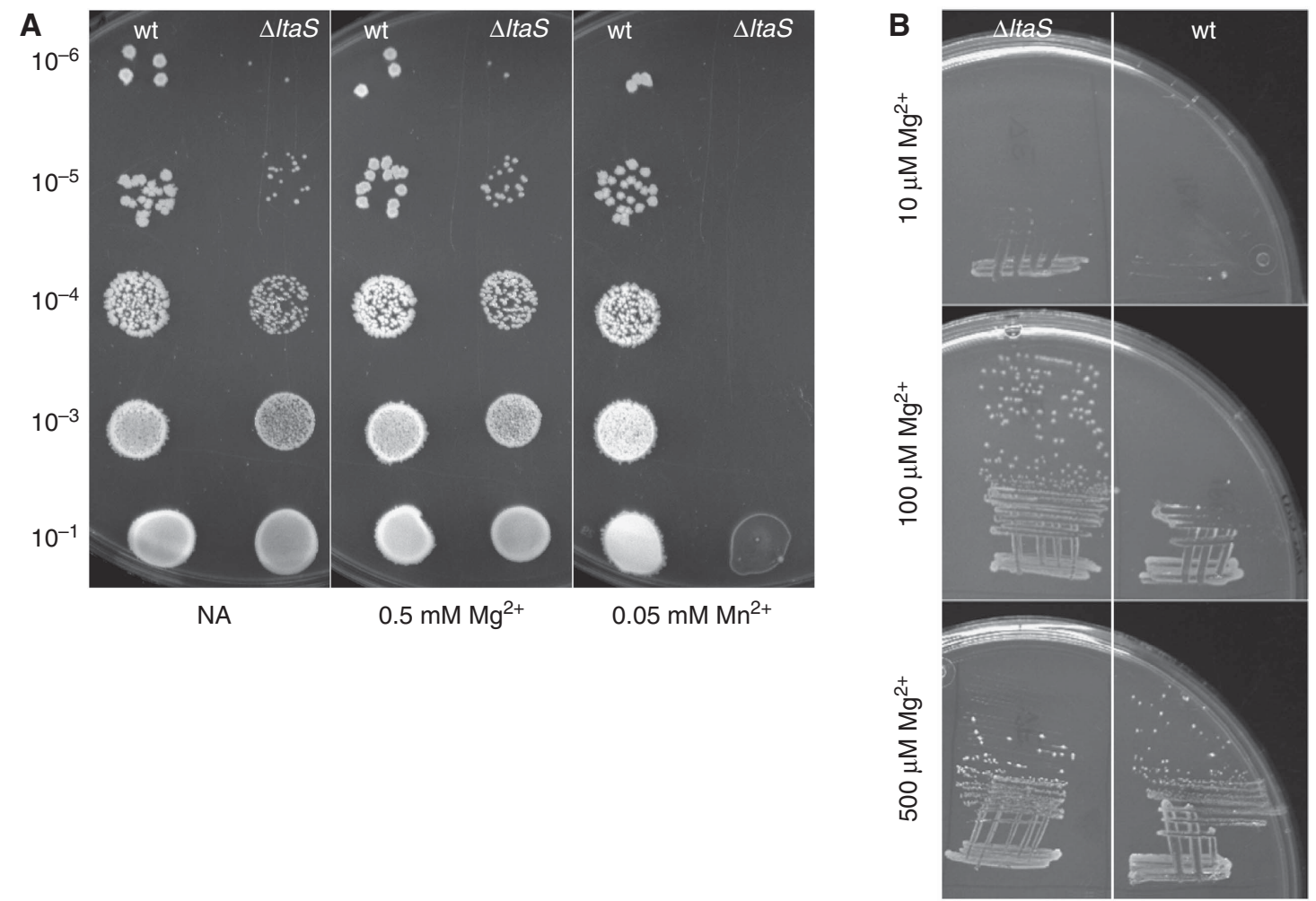

Figure 2 Effect of metal ion concentration on the viability of wild type and ltaS mutants. (A) Growth of wild type (168) and ltaS mutant (strain 4286) on NA plates (left panel) containing $0.05 \mathrm{mM} \mathrm{Mg}^{2+}$ (middle panel) or $0.5 \mathrm{mM} \mathrm{Mn}^{2+}$ (right panel). The strains were grown to midexponential phase and spotted onto the plates in the dilutions as indicated. (B) Growth of ltaS mutant (strain 4283, left) and wild type (strain 168 , right) on minimal medium plates containing 10,100 and $500 \mu \mathrm{M} \mathrm{Mg}^{2+}$ as indicated. 
Table I Effects of mutations in lta genes on cell diameter

\begin{tabular}{|c|c|c|c|}
\hline Strain & Genotype & Average cell diameter $(\mu \mathrm{m}) \pm$ s.d. & Range $(\mu \mathrm{m})$ \\
\hline 168 & wt & $0.85 \pm 0.07$ & $0.67-1.04$ \\
\hline 4283 & $\Delta l t a S$ & $0.75 \pm 0.08$ & $0.57-0.98$ \\
\hline 4287 & $\Delta y f n I$ & $0.84 \pm 0.06$ & $0.66-1.02$ \\
\hline 4292 & $\Delta y q g S$ & $0.84 \pm 0.05$ & $0.67-0.99$ \\
\hline 4295 & syvg & $0.87 \pm 0.04$ & $0.73-0.98$ \\
\hline 4610 & $\Delta l t a s \Delta y f n I$ & $0.78 \pm 0.09$ & $0.54-1.10$ \\
\hline 4611 & $\Delta l t a S \Delta y q g S$ & $0.76 \pm 0.07$ & $0.54-0.96$ \\
\hline 4612 & $\Delta l t a S \Delta y v g J$ & $0.74 \pm 0.05$ & $0.60-0.93$ \\
\hline 4613 & $\Delta y f n I$ syqgS $\Delta y v g J$ & $0.81 \pm 0.06$ & $0.68-1.00$ \\
\hline 4620 & $\Delta l t a S$ syfnI $\Delta y q g S$ syvgJ & $0.85 \pm 0.09$ & $0.66-1.16$ \\
\hline 4298 & $\Delta l t a S \Delta m b l$ & $0.80 \pm 0.06$ & $0.67-0.97$ \\
\hline
\end{tabular}

Cells were grown in PAB medium to exponential phase, stained with Nile Red, and cell width was measured using ImageJ.
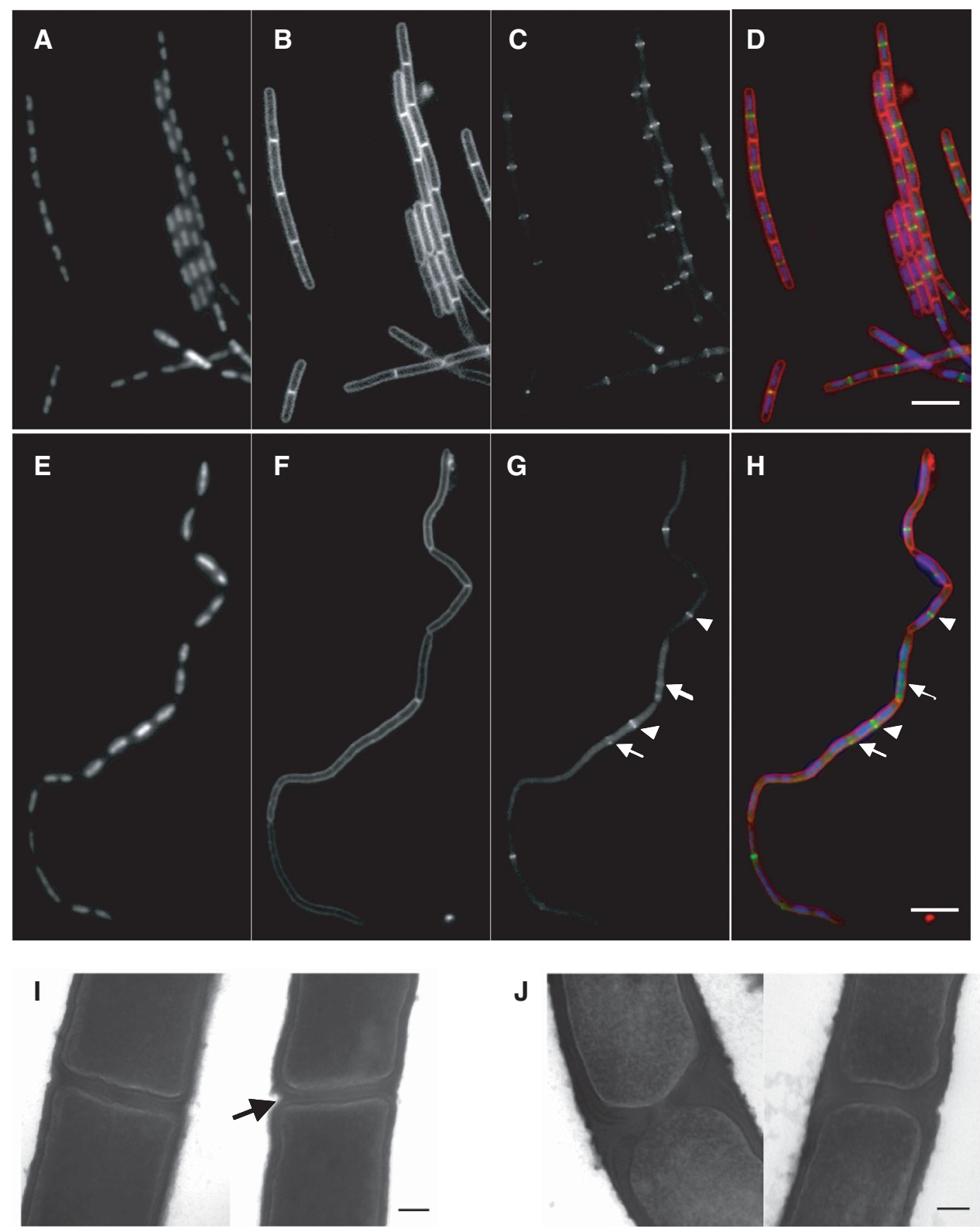

Figure 3 Effects of ltaS mutation on FtsZ ring formation, cell division and cell separation. The strains were grown to mid-exponential phase in $\mathrm{PAB}$ medium at $30^{\circ} \mathrm{C}(\mathbf{A}-\mathbf{H})$ or $37^{\circ} \mathrm{C}(\mathbf{I}, \mathbf{J})$. (A-H) Samples of wild type (strain 2020; A-D) and ltaS mutant (4605; E-H) carrying gfp-ftsZ under a xylose-inducible promoter were stained with DAPI (for DNA) and FM5-95 (for the membrane), and additionally imaged for FtsZ-GFP localization. (D, H) Overlays of the membrane (red), DNA (blue) and GFP (green) signals. Scale bar $5 \mu \mathrm{m}$. (I, J) Transmission electron microscopic images of transverse sections of two representative septa each of wild type (168; I) and ltaS mutant (strain 4284; J). Scale bar $100 \mathrm{~nm}$. 
intervals in the chains (compare images of wild type and mutant in Figure 3B and F). Interestingly, the effect on division was exerted at an early step in the process. Assembly of FtsZ protein into a ring at sites of impending division is the earliest marker for cell division (reviewed by Dajkovic and Lutkenhaus, 2006). In wild-type cells, bands (which correspond to rings seen edge-on) of FtsZ-GFP are formed at regular positions between segregating nucleoids (Figure 3C). In contrast, FtsZ-GFP localization was deranged in the ltaS mutant (Figure 3G). When clear bands of FtsZGFP were observed, they were usually correctly positioned between well-separated nucleoids (arrowheads). However, FtsZ-GFP bands were either missing or only present as faint bands (arrows) at many potential division sites, and there appeared to be a higher than normal background of non-localized fluorescence. DAPI staining showed that chromosomes were replicating and segregating more or less normally in the mutant, suggesting that the division defect was not secondary to a chromosome segregation defect (Figure $3 \mathrm{~A}$ and $\mathrm{E}$ ).

Transmission electron microscopy (TEM) revealed that the morphology of the division septa formed in ltaS mutants was abnormal. In the typical examples shown in Figure 3I and J, the wild-type septum was of fairly uniform thickness and the membranes at the nascent sister cell poles were rather flattened, curved only near their outer edges at the junction with the lateral wall (Figure 3I). In contrast, the mutant septa were abnormally thick, especially towards their outer edges, with unusual triangular wedges of wall material (Figure 3J). Furthermore, it appeared that the autolytic activity that leads eventually to cell separation (Blackman et al, 1998) and which usually begins at the outer edges of the septa (arrowed for the wild-type cell in Figure 3I), was delayed in the ltaS mutant. Autolytic enzyme activity has previously been suggested to be regulated by anionic polymers and the ionic environment of the cell wall (Cheung and Freese, 1985; Wecke et al, 1997; Smith et al, 2000). ltaS mutants therefore have important defects in both formation of the cell division septum and subsequent cell separation. We suggest that these defects occur, at least in part, because the impaired cation homoeostasis interferes with proper regulation of the activities of the enzymes that carry out the synthesis and turnover of the wall at division sites.

\section{Partial functional redundancy of LtaSs in B. subtilis}

A most likely explanation for the viability of the single ltaS mutant was that $B$. subtilis has three paralogues of ltaS, namely $y f n I, y q g S$ and $y v g J$. These genes encode products predicted to be about 53, 44 and $41 \%$ identical to LtaS, respectively (based on BLAST searches run on the SubtiList web server (Moszer et al, 2002). Only expression of ltaS, however, restored LTA synthesis in an S. aureus ltaS deletion strain (Gründling and Schneewind, 2007b). We constructed null mutants for each of the three paralogues and examined their microscopic and growth phenotypes. None of the single mutants had a discernible effect on growth rate or cell morphology (Supplementary Figure 1B, D-H). Combinations of mutations were then made. All double mutants combining with ltaS were comparable in growth and morphology to the single ltaS mutant, and a triple $\Delta y f n I$ syqgS $\Delta y v g J$ mutant did not differ noticeably from the wild type (data not shown).
Surprisingly, we were readily able to construct a strain in which all four ltaS paralogues were disrupted, indicating that, in contrast to $S$. aureus, LTA is not essential in B. subtilis. However, the phenotype of the quadruple mutant $\Delta l t a S \Delta y f n I$ $\Delta y q g S \Delta y v g J$ was more severe than that of the ltaS single mutant: the mutant strain grew very slowly with a doubling time of about $48 \mathrm{~min}$ in $\mathrm{PAB}$ medium at $37^{\circ} \mathrm{C}$, whereas the doubling time of the ltaS single mutant was around $31 \mathrm{~min}$, and the wild type doubled every $26 \mathrm{~min}$ under the same conditions (Supplementary Figure 1C). The quadruple mutant also had a severe cell division and cell separation defect, resulting in the formation of filamentous clumps of cells; the cell filaments were tightly twisted around their long axis and lysis of cell compartments was common (Supplementary Figure 1I). Taken together, these observations support the idea of a partial functional redundancy of the four LtaS-like proteins.

Interestingly, the coiling and clumping of the strain lacking LTA were reminiscent of the $m b l$ mutant phenotype. We suggest, that in both strains an imbalance of processes involved in cell wall synthesis or modifications of the electrostatic properties of the wall lead to twisting and macrofibre formation (Mendelson et al, 1985).

Analysis of the expression of these genes through fusions to $l a c Z$ revealed that $l t a S$ was expressed much more strongly than the other genes under normal growth conditions (Supplementary Figure 1A). In previous proteomic studies (Hirose et al, 2000), LtaS and YfnI, but neither YqgS nor YvgJ, were detected in growing cells. Transcription of $y f n I$ is dependent on the extracytoplasmic sigma factor $\sigma^{\mathrm{M}}$ (Jervis et al, 2007), which is involved in salt stress resistance. However, we did not detect any effect of salt stress on the $y f n I$ single mutant, nor on the ltaS yfnI double mutant (not shown).

These results indicate that LtaS is the major LTA synthase, whereas the other three paralogues have a minor function and might be required for adaptation to specific environmental or developmental conditions.

\section{Specific and essential requirement for LtaS during stages II-III of sporulation}

Although the four single mutants ( $\Delta l t a S, \Delta y f n I, \Delta y q g S$ and $\Delta y v g J$ ) were sporulation proficient, we were surprised to find that the ltaS yqgS double mutant did not form spores, as

Table II Effects of lta mutations on sporulation frequency

\begin{tabular}{|c|c|c|}
\hline Strain & Genotype & $\begin{array}{c}\% \text { sporulation } \\
\text { (no. of cells counted) }\end{array}$ \\
\hline 168 & wt & $57(2415)$ \\
\hline 4283 & $\Delta l t a S$ & $47(1425)$ \\
\hline 4287 & $\Delta y f n I$ & 57 (2719) \\
\hline 4292 & $\Delta y q g S$ & $56(3796)$ \\
\hline 4295 & syvgJ & $61(1972)$ \\
\hline 4610 & $\Delta l t a S \Delta y f n I$ & 41 (2719) \\
\hline 4611 & $\Delta l t a S \Delta y g g S$ & $<0.07(1536)$ \\
\hline 4612 & $\Delta l t a S \Delta y v g J$ & $2.1(3676)$ \\
\hline 4613 & $\Delta y f n I$ syqgS $\Delta y v g J$ & $45(4043)$ \\
\hline 4627 & $\Delta$ ltas $\Delta y q g S P_{x y l}$ gfp-ltas & 43 (2083) \\
\hline 4628 & $\Delta$ ltaS $\Delta y q g S P_{x y l} g f p$-ltaS T297A & $<0.03(3578)$ \\
\hline
\end{tabular}

Cells were grown in Schaeffer's medium for $24 \mathrm{~h}$, and then sporulation efficiency was determined by microscopic counting. 
judged by phase-contrast microscopy (Table II). In contrast, the other double mutants in combination with ltaS did sporulate, although the ltaS yvgJ mutant had a reduced efficiency (Table II). The triple mutant $\Delta y f n I \Delta y q g S \Delta y v g J$ sporulated almost similar to the wild type. Remarkably, when a more sensitive method was used to measure the spore frequency (numbers of heat- or chloroform-resistant spores formed in liquid culture), spores could not be detected for the ltaS yqgS double mutant $\left(<10^{-7}\right.$ spores per viable cell; under the same conditions we observed over 0.6 spores per viable cell for the ltaS single mutant). Such a severe sporulation block is normally associated only with mutations that eliminate key regulatory proteins or proteins involved in critical morphological or structural changes that occur during spore development (Piggot and Coote, 1976; Errington, 1993). These results suggested that LTA is essential for sporulation and that expression of the minor LtaS, yqgS, provides sufficient activity to compensate for loss of the major LtaS, ltaS.
Disruption of both genes results in the severe sporulation defect.

Preliminary microscopic examination suggested that sporulation was arrested at stage II, when a division septum is formed near one pole of the cell. Activation of a key transcription factor, the sigma factor, $\sigma^{\mathrm{F}}$, is dependent on the formation of the septum (reviewed by Errington, 1993; Hilbert and Piggot, 2004), so we tested whether the synthesis and activation of $\sigma^{\mathrm{F}}$ were affected by ltaS yqgS disruption. lacZ and mCherry fusions to the spoIIA promoter, which drives expression of the operon that encodes $\sigma^{\mathrm{F}}$, showed that spoIIA was efficiently activated to similar levels as in the ltaS single mutant (Figure $4 \mathrm{~A}$ and data not shown). In contrast, expression of a lac $Z$ reporter gene fusion to a promoter that is dependent on active $\sigma^{\mathrm{F}}$ (that of spoIIQ) was completely blocked in the double mutant (Figure 4B). Similarly, the natural reporter enzyme alkaline phosphatase (AP), which depends on $\sigma^{\mathrm{F}}$ indirectly through activation of
A

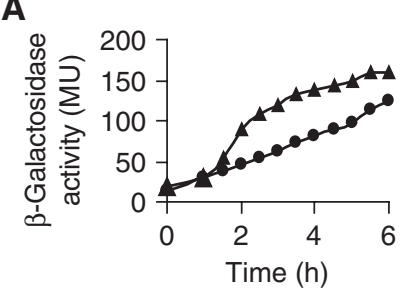

B

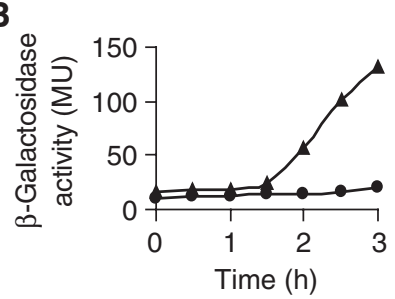

C

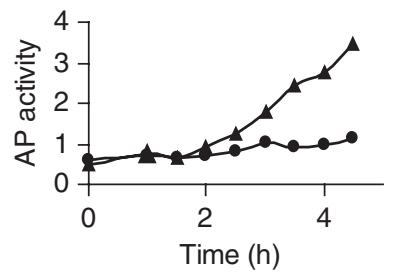

D

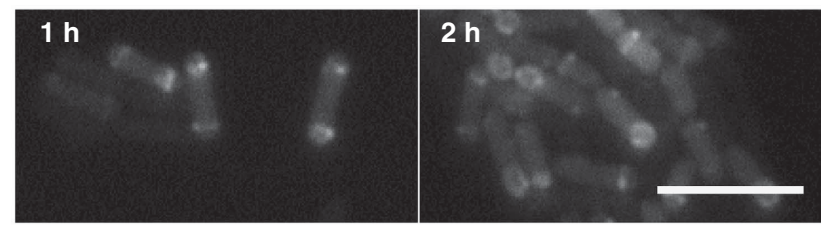

E
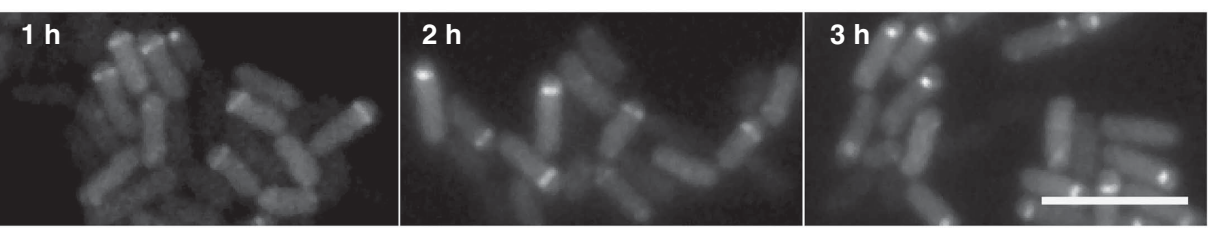

$\mathbf{F}$

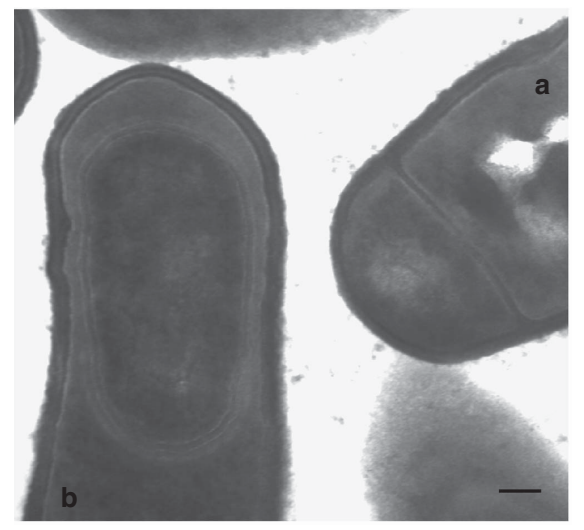

G

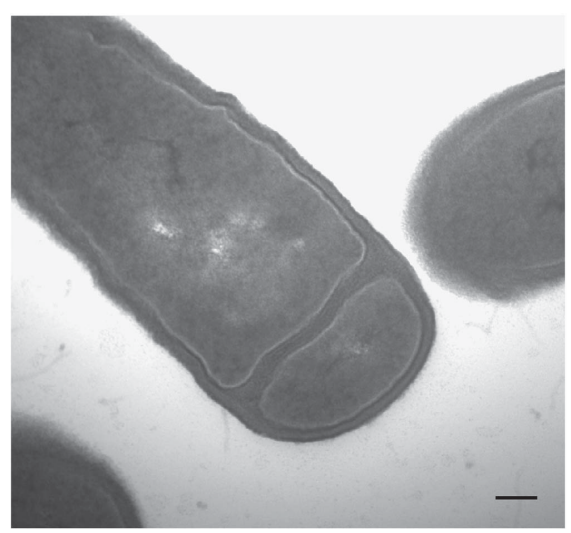

Figure 4 Effects of ltaS and yqgS deletion on sporulation. (A) Expression pattern of the spoIIA promoter measured by assaying $\beta$-galactosidase activity of strains 4614 (ltaS mutant, $\mathbf{\Lambda}$ ) and 4615 (ltaS yqgS double mutant, $\mathbf{O}$ ) grown in Schaeffer's medium at $37^{\circ} \mathrm{C}$. (B) Measurements of $\beta$-galactosidase activity of strains ltaS mutants $(4616, \boldsymbol{\Delta})$ and ltaS yqgS double mutants $(4617, \boldsymbol{0})$ carrying the lacZ gene under control of the $\sigma^{\mathrm{F}}$-inducible spoIIQ promoter. (C) Assay for sporulation-specific alkaline phosphatase activity in ltaS mutant (4283, $\left.\mathbf{\Delta}\right)$ and ltaS yqgS double mutant $(4611, \mathbf{O})$. All strains were grown in Schaeffer's medium at $37^{\circ} \mathrm{C}$, time point 0 was set at the entry into stationary phase (A-C). (D, E) Localization of SpoIIE-GFP in an ltaS mutant background (strain 4618 (D)) and an ltaS yqgS double-mutant background (strain 4619 (E)). Time was measured from the point at which fluorescence of mCherry (under control of the spoIIA promoter) first became visible and images were taken at the time points indicated. Scale bar $5 \mu \mathrm{m}$. (F, G) Transmission electron microscopic images of sporulating cultures of ltaS mutant (4284 (F); asymmetric septum is marked 'a', engulfed forespore is marked 'b') and ltaS yqgS double mutant (4611 (G)). The strains were grown for $9 \mathrm{~h}$ in Schaeffer's medium at 37 before fixation. Scale bar $100 \mathrm{~nm}$. 
$\sigma^{\mathrm{E}}$ (Glenn and Mandelstam, 1971; Piggot and Coote, 1976; Stragier et al, 1984; Illing and Errington, 1991) was also blocked by the ltaS yqgS double deletion (Figure 4C). This effect was not due to a failure in translocation of the prespore chromosome (Wu and Errington, 1994), based on (i) DAPI staining (data not shown) and (ii) the fact that the spoIIQlacZ fusion was located at $a m y E$, which is captured in the prespore compartment even when chromosome translocation fails (Wu and Errington, 1994).

A key player in the machinery regulating $\sigma^{\mathrm{F}}$ activation is SpoIIE. This protein has a dual role in sporulation, being required for proper formation of the polar septum and for the correct spatial and temporal activation of $\sigma^{\mathrm{F}}$ (reviewed by Errington, 2003; Hilbert and Piggot, 2004). Expression of spoIIE is turned on during the early stages of sporulation (stage 0) and its product is targeted to the polar division sites (Arigoni et al, 1995; Barak et al, 1996; Wu and Errington, 1998). We therefore examined the effects of the ltaS yqgS deletion on synthesis and targeting of SpoIIE through a spoIIE-gfp fusion. As expected, SpoIIE-GFP was expressed in both single and double-mutant cells (Figure 4D and E). The SpoIIE-GFP fusion also appeared to target to the polar septa that were formed in the double-mutant cells as well as in the single mutant. However, in the ltaS yqgS double mutant, SpoIIE-GFP remained static at the asymmetric septa (Figure 4E), whereas in the ltaS single mutant it progressed rapidly to the next step of spore development, engulfment of the prespore (ovoid GFP signals in the $2 \mathrm{~h}$ image in Figure 4D). Taken together, these results suggest that the block in sporulation occurs in the signalling pathway for $\sigma^{\mathrm{F}}$ activation, but downstream of SpoIIE synthesis and recruitment to the polar septum.

As $\sigma^{\mathrm{F}}$ activation is thought to be coupled to polar septation and vegetative division septa were abnormal in the ltaS single mutant, we questioned whether the double mutant might be affected in the ultrastructure of the polar septum. TEM images of culture samples taken $4 \mathrm{~h}$ after initiation of sporulation showed that the ltaS single mutant formed apparently normal, thin asymmetric septa, similar to those of wild-type cells (cell marked 'a' in Figure 4F) (Hilbert and Piggot, 2004). We also noticed that many cells went on to engulf the prespore in a typical manner (Figure 4F, cell 'b'). In contrast, the septa of the ltaS yqgS double mutant were highly abnormal. In the typical example shown (Figure 4G), the septum was much thicker than normal, almost the same as in vegetative cells (compare to Figure 3I), and is reminiscent of the abnormal septa of spoIIE mutant cells (Illing and Errington, 1991; Khvorova et al, 1998; Carniol et al, 2005) or a divIB mutant (Thompson et al, 2006). The presence of highly abnormal polar septa in the double mutant, but not in the ltaS single mutant, provided a likely explanation for the failure in $\sigma^{\mathrm{F}}$ activation and suggests that LTA is a key component in the polar sporulation septum upon which the pivotal cell-specific $\sigma^{\mathrm{F}}$ activation mechanism is strictly dependant. Therefore, these results point to an important role for LTA synthesis in cell division of both vegetative and sporulating cells. The results also suggest that the ltaS paralogues contribute to LTA function differently, with yfnI supporting ltaS function during vegetative growth and yqgS and $y v g J$ acting in stationary phase. The molecular basis for the block in $\sigma^{\mathrm{F}}$ activation remains to be resolved.

\section{LtaS and YqgS localize predominantly at division sites in both vegetative and sporulating cells}

The results described above suggest an important role for LTA in cell division, of both vegetative and sporulating cells. If true, the synthases ought to be localized at division sites, at least in part. A range of predictive bioinformatic tools, such as TMpred (Hofmann and Stoffel, 1993) predict that LtaS and its paralogues have five transmembrane domains near their $\mathrm{N}$ termini, followed by a large C-terminal extracellular domain, which contains the catalytic centre responsible for poly(GroP) synthesis (see below). GFP fusions to LtaS and YqgS were at least partially functional as judged by restoration of wild-type growth rate and cell shape (GFP-LtaS) and sporulation proficiency (both fusions expressed in the appropriate deletion backgrounds). Although the fusions gave relatively weak fluorescence signals, they were clearly associated with the cell periphery. Importantly, both proteins appeared to be enriched at division sites, in both vegetative and sporulating cells (Figure 5A and B). As the doublemutant cells produced septa that resembled those of spoIIE mutants (see above) it was possible that the SpoIIE phenotype was due to delocalized YqgS. However, GFP-YqgS still localized to polar septa in a spoIIE deletion background (Figure 5C). Indeed, fluorescence was often clearly visible at polar septa near both cell poles; a phenotype characteristic of spoIIE mutants (Piggot and Coote, 1976; Illing and Errington, 1991). These results support the idea that LTA synthesis is particularly important for cell division.
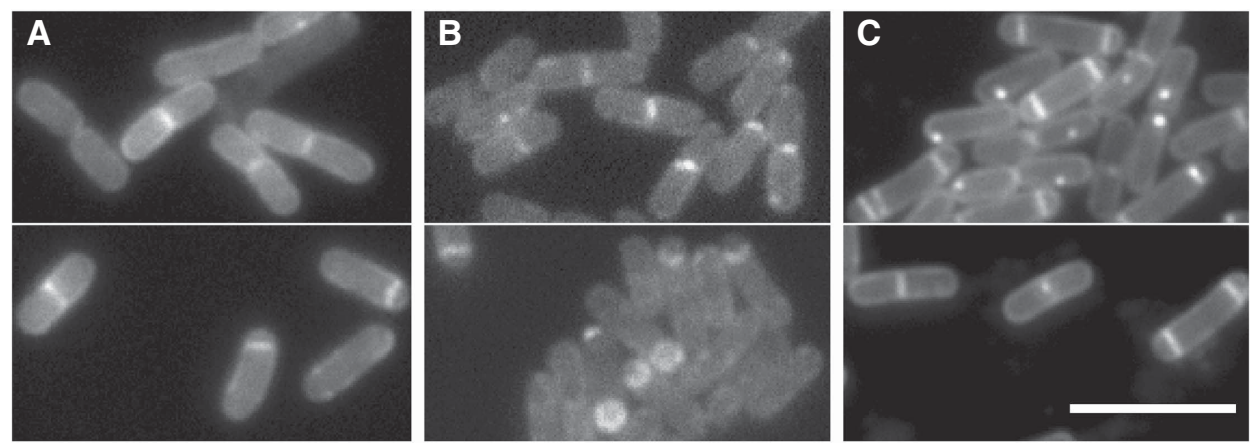

Figure 5 GFP-LtaS and GFP-YqgS localization. Fluorescence microscopy of strains 4607 and 4609 carrying inducible alleles of $g f p$-ltaS (A) and gfp-yqgS (B) at the amyE locus. Localization of GFP-YqgS in an spoIIE mutant background (strain 4626 (C)). Of each strain two fields are shown, all strains were grown in S-medium containing $0.5 \%$ xylose at $30^{\circ} \mathrm{C}$. Scale bar $5 \mu \mathrm{m}$. 


\section{Evidence that LTA and WTA have specialized functions in cell morphogenesis}

The phenotype of cells devoid of LTA differs clearly from that previously reported for deficiency in WTA. The latter mutants, for example in tagO or tagF, typically become bloated and spherical (Wagner and Stewart, 1991; D'Elia et al, 2006a), but are still capable of division, as can many other mutants with a specific defect in cell elongation (Henriques et al, 1998; Wei et al, 2003; Leaver and Errington, 2005). Consistent with this role, the localization of several Tag proteins, particularly TagG and TagH, suggests that they might be associated with the cell elongation machinery (Formstone et al, 2008). In contrast, the above results showed that LTA-deficient mutants have a distinct and almost complementary phenotype, in efficiently elongating along a longitudinal growth axis (albeit with a considerable level of twisting) but clearly impaired in cell separation and cell division.

We were now in a position to test the effects of loss of both TA systems in B. subtilis. First, we introduced a deletion in the major LtaS gene, ltaS, into a tagO deletion strain. The resultant strain was viable and phenotypically resembled the tagO single mutant in being rounded and growing in clumps (Figure 6B; compare with Figure 6A) (D'Elia et al, 2006a). Attempts to combine all four ltaS mutations and tagO failed, suggesting that this combination might be lethal. We therefore constructed a strain in which ltaS was controlled by a xylose-inducible promoter. We then deleted the other three ltaS paralogues, and were able to introduce the $\operatorname{tagO}$ deletion in the presence of xylose. Results shown in Figure $6 \mathrm{E}$ confirmed that inactivation of both the LTA and WTA systems was indeed lethal. The $P_{x y l}$-ltaS strain (4622) grew well in the presence or absence of xylose. Combined with the triple deletion of the other ltaS paralogues (strain 4623), growth was still reasonable, though slightly reduced in the absence of xylose. The tagO $P_{x y l}$-ltaS mutant (strain 4624) grew poorly on both the xylose and non-xylose plates, but when the triple deletion was also present (strain 4625), growth in the absence of xylose was abolished. The two tagO $P_{x y l}$-ltaS-containing mutants (with and without the triple deletion) were then cultured in liquid PAB medium $\left(+\mathrm{Mg}^{2+}\right)$ with and without xylose (Figure 6F). The growth rate of the tagO $P_{x y l}$ ltaS mutant was reduced but still detectable in the absence of xylose (open triangles), but the pentuple mutant failed to grow at all in the absence of inducer (open circles). Figure 6C shows that the morphological appearance of the pentuple mutant in the presence of xylose was similar to that of the other tagO mutants, except that the cells were somewhat larger and even more clumped. Cells cultured for several hours in the absence of xylose showed little differences in morphology, suggesting that growth was arrested without any further change in morphology (Figure 6D). The lethality arising from loss of both TA systems shows that synthesis of anionic polymers is essential for B. subtilis. There are two ways in which this lethality might arise, which we cannot yet distinguish. The first possibility is that complete loss of anionic polymer synthesis affects divalent cation homoeo-
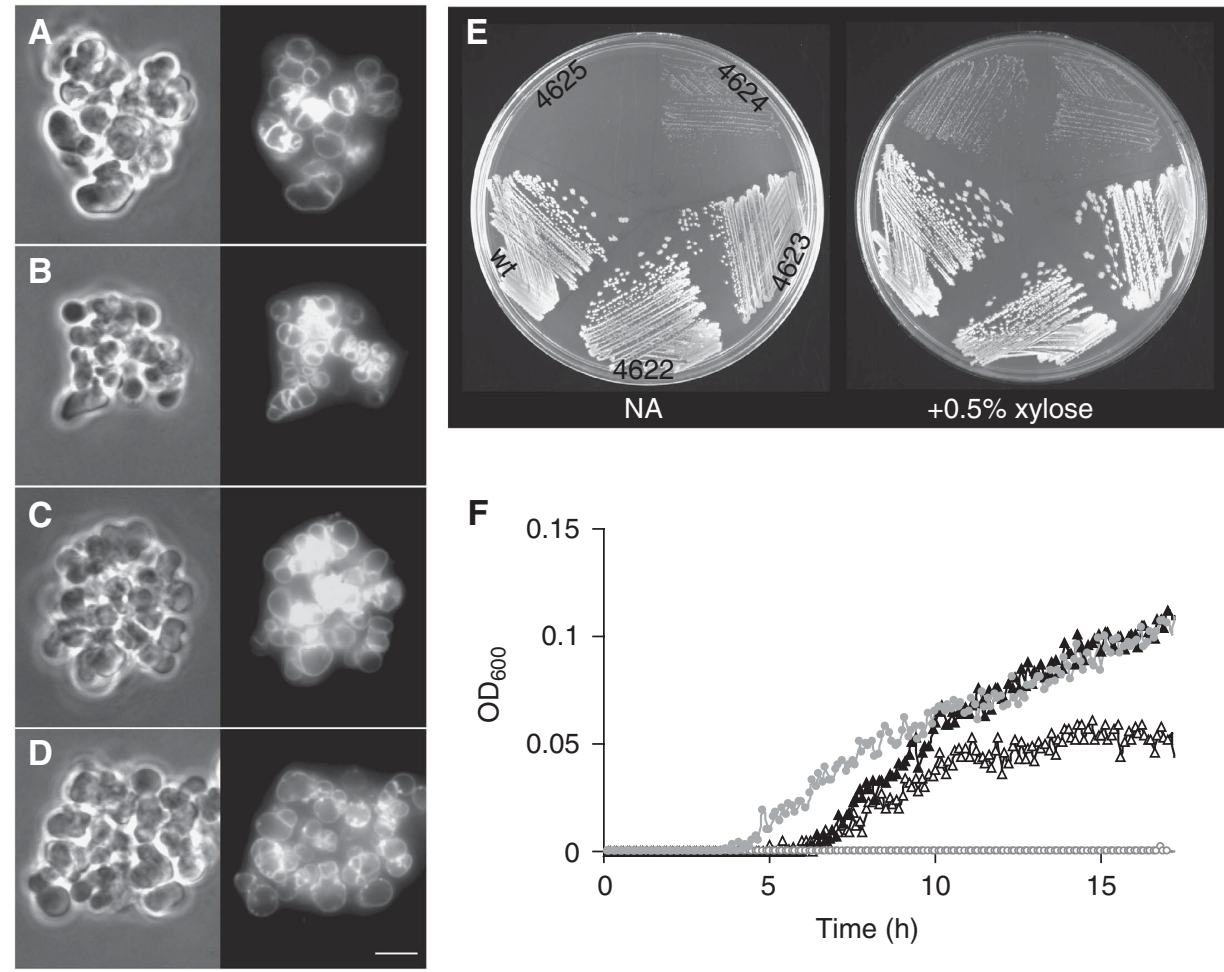

Figure 6 Morphology and viability of LTA and WTA mutants. (A-D) Morphology of a tagO mutant (strain 4282 (A)) and an ltaS tagO double mutant (strain 4621 (B)). Strain 4625 growing in the presence of $0.5 \%$ xylose (C) and after depleting for xylose (D). Left panels show phasecontrast microscopic images, right panels show staining of the membrane with FM5-95. The strains were grown in PAB medium with the addition of $10 \mathrm{mM} \mathrm{Mg}^{2+}$ at $37^{\circ} \mathrm{C}$, scale bar $5 \mu \mathrm{m}$. (E) Strains $4622\left(\mathrm{P}_{x y l}\right.$ ltaS), 4623 ( $\Delta y$ fnI $\Delta y q g S \Delta y v g J P_{x y l}$ ltaS), $4624\left(\Delta t a g O P_{x y l}\right.$ ltaS), 4625 ( $\Delta$ tagO $\Delta y f n I \Delta y q g S \Delta y v g J P_{x y l}$ ltaS) and wild type (168) were cultured on NA with (right) or without $0.5 \%$ xylose (left) overnight at $37^{\circ} \mathrm{C}$. (F) Growth curve of strains $4624\left(\Delta\right.$ tagO $P_{x y l}$ ltaS, $\left.\mathbf{\Delta}\right)$ and 4625 ( $\Delta$ tagO $\Delta y f n I \Delta y q g S \Delta y v g J P_{x y l}-$ ltaS, ) in PAB medium containing $10 \mathrm{mM} \mathrm{Mg}{ }^{2+}$ with (closed symbols) or without (open symbols) addition of $0.5 \%$ xylose. The cultures were grown in a microtitre plate while shaking at $37^{\circ} \mathrm{C}$. 
stasis so badly that multiple enzyme systems are inhibited resulting in growth arrest. The second is that impairment of both the elongation- and division-associated wall synthetic systems eliminates all routes to growth.

\section{Crystal structure of LtaS $_{215-649}$}

LtaS has an important function in the morphology and physiology of $B$. subtilis. To gain insights into the enzymatic function performed by LtaS, we solved the structure of the extracellular domain (residues 215-649, $\mathrm{LtaS}_{215-649}$ ) by X-ray crystallography using a selenomethionine-substituted sample and anomalous scattering procedures. The experimental SAD electron density maps were of such quality that the entire structure of the domain, bar the last 14 amino acids, could be built and refined to convergence. The two independent molecules in the asymmetric unit are indistinguishable and can be considered equal. Details of the data collection, structure solution and refinement are in Table III.

Globally, $\mathrm{LtaS}_{215-649}$ belongs to the AP superfamily (Millán, 2006), despite sharing only 15\% sequence identity to its closest structural neighbours in the PDB (Figure 7A). The core of $\mathrm{LtaS}_{215-649}$ is an $\alpha / \beta$-fold, common to all its structural homologues. The nearest homologue is an unpublished output from a structural genomics effort (PDBid 3ed4), which is annotated as a putative arylsulphatase from Escherichia coli. The two structures can be superimposed which an r.m.s.d. on 310 matched $\mathrm{C}_{\alpha}$ atoms of $2.8 \AA$. The AP superfamily also comprises eukaryotic and prokaryotic sulphatases, as well as phosphodiesterases, mutases and dehydratases, examples of which are all found by SSM in a search of the PDB for structural homologues of $\mathrm{LtaS}_{215-649}$.

Table III Crystallographic data collection

\begin{tabular}{|c|c|}
\hline \multicolumn{2}{|l|}{ Data collection } \\
\hline Wavelength $(\AA)$ & 0.9763 \\
\hline Space group & $P 12_{1} 1$ \\
\hline Unit cell parameters & $\begin{aligned} a & =56.63 \AA, b=54.41 \AA \\
c & =140.78 \AA, \beta=90.97^{\circ}\end{aligned}$ \\
\hline Resolution $(\AA)$ & $70.36-2.35(2.48-2.35)$ \\
\hline No. of observations & 704196 \\
\hline No. of unique reflections & 35957 \\
\hline Multiplicity & $19.6(7.7)$ \\
\hline Anomalous multiplicity & $10.1(3.9)$ \\
\hline Completeness (\%) & $100(100)$ \\
\hline Anomalous completeness & $100(100)$ \\
\hline Average $I /$ sigma $I$ & $22.1(3.8)$ \\
\hline$R_{\text {sym }}$ & $0.124(0.361)$ \\
\hline \multicolumn{2}{|l|}{ Refinement } \\
\hline No. of reflections & 34148 \\
\hline$R_{\text {work }}(\%)$ & $17.1(21.2)$ \\
\hline$R_{\text {free }}(\%)$ & $23.3(29.9)$ \\
\hline No. of atoms & 7080 \\
\hline \multicolumn{2}{|l|}{ r.m.s.d. } \\
\hline Bond lengths $(\AA)$ & 0.012 \\
\hline Bond angles (deg) & 1.382 \\
\hline \multicolumn{2}{|l|}{ Ramachandran plot } \\
\hline Favoured (\%) & 96.1 \\
\hline Allowed (\%) & 99.9 \\
\hline \multicolumn{2}{|l|}{ Mean B-factor } \\
\hline Wilson B $\left(\AA^{3}\right)$ & 39.5 \\
\hline Main chain $\left(\AA^{3}\right)$ & 48.3 \\
\hline Side chain/water $\left(\AA^{3}\right)$ & 47.3 \\
\hline
\end{tabular}

The overall similarity of LtaS $_{215-649}$ to biochemically characterized enzymes enables the location of the active centre of LtaS to be deduced. First, it was clear immediately on solving the structure that Thr297 is phosphorylated in the crystal (Figure 7; Supplementary Figure 2A). This feature has been refined satisfactorily with unit occupancy and with B-factors similar to nearby, but non-bonded atoms. Second, there is a single metal ion found adjacent to the phosphoryl group that was modelled and refined satisfactorily as magnesium. This metal ion was also present in the crystallization conditions. These electron density features allowed the identification of the active site of LtaS, which resides in the centre of the $\alpha / \beta$ domain, with Thr297 situated at the N-terminal end of the central $\alpha$-helix. The active site is in a cleft on the surface that, in the context of the full-length native protein, would oppose the cell membrane (this is depicted schematically in Figure 7C), the source of phosphatidyl glycerol for LTA synthesis.

To test whether Thr297 was important for LtaS function, we made a mutation that would generate a Thr297Ala substitution. Purification and analysis of the mutant protein showed that phosphorylation was prevented (only one peak at $49.71 \mathrm{kDa}$, the expected mass for the protein carrying the point mutation). Western blotting of mutant B. subtilis cells showed that the protein accumulated to similar levels as the wild-type protein (data not shown). Functionality was assayed by examining the sporulation efficiency when the protein carrying the point mutation was expressed in an otherwise sporulation-deficient ltaS yqgS double deletion strain (Table II). The mutant protein was not able to rescue the sporulation-deficient phenotype. We therefore conclude the phosphorylatable Thr297 is essential for LtaS function.

Sequence analysis of the amino acids that are in direct contact with the phosphothreonine at position 297 (His412, Glu253 and Trp350) revealed that they are among the most conserved in LtaS orthologues, suggesting their importance for LTA synthesis. The single magnesium ion in LtaS lies in a distorted octagonal coordination geometry (Figure 7B), coordinated in the equatorial plane by the N $\varepsilon 2$ atom of His472, the ether oxygen of phospho-Thr297, and in a bidentate manner by the carboxylate of Glu253, whereas the apical positions are filled by O $\delta 2$ of Asp471 and O1P of phosphoThr297. A single metal ion is also observed in structures of arylsulphatases (Boltes et al, 2001), whereas AP contains three metal ions, only two of which (both zinc) appear to be catalytically active and which straddle the phosphate in a non-covalent enzyme: $P_{i}$ complexes (reviewed in Galperin and Jedrzejas, 2001). The second of these zinc ions-equivalent in position to the magnesium in LtaS-also coordinates the ether oxygen of the phosphoserine, and is involved in the hydrolysis of the phosphoseryl intermediate (Galperin and Jedrzejas, 2001).

The arylsulphatase group of enzymes are characterized by the unusual requirement for the post-translational formylation of an invariant cysteine, forming C $\alpha$-hydroxyformylglycine, to become the active site nucleophile (e.g., Jonas et al, 2008). As has been described for alkaline phosphatases, the reaction cycle of the sulphatase subfamily of the AP superfamily also proceeds through a covalent enzyme intermediate-here the nucleophilic C $\alpha$-hydroxyformylglycine becomes sulphated (e.g., lysosomal sulphatase; Bond et al, 1997). Phospho-Thr297 is spatially equivalent to the 
A
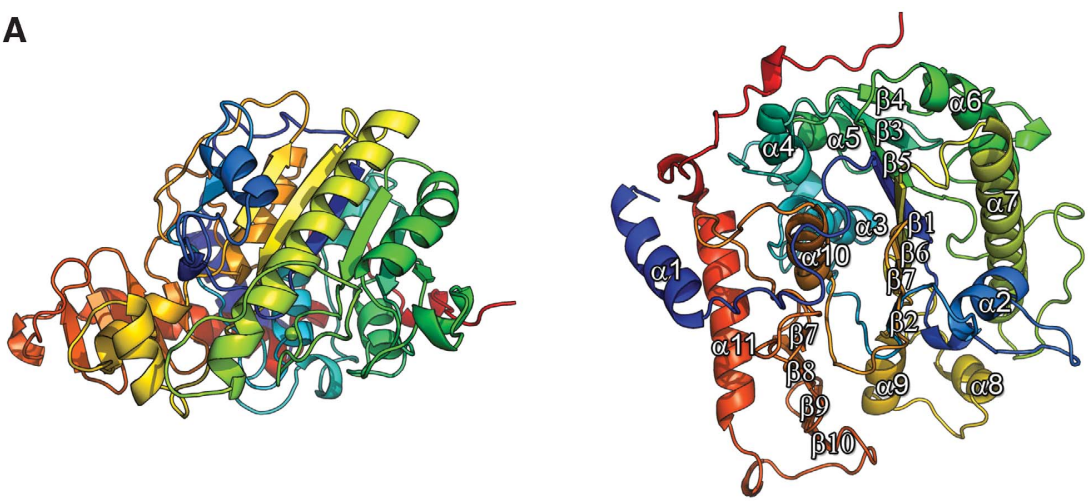

B

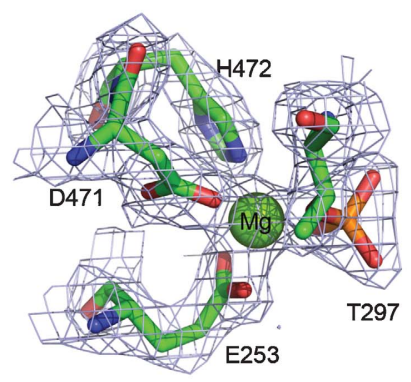

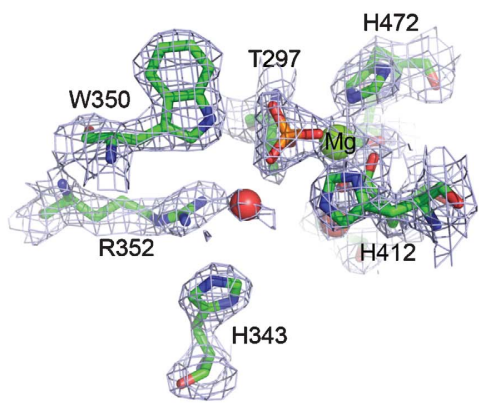

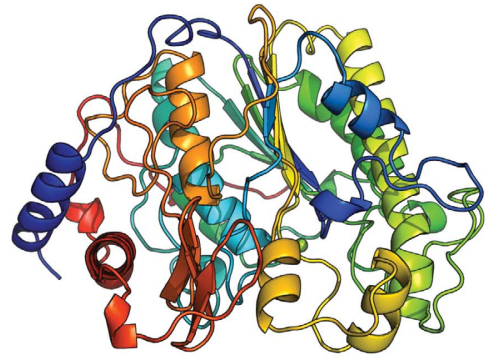

C

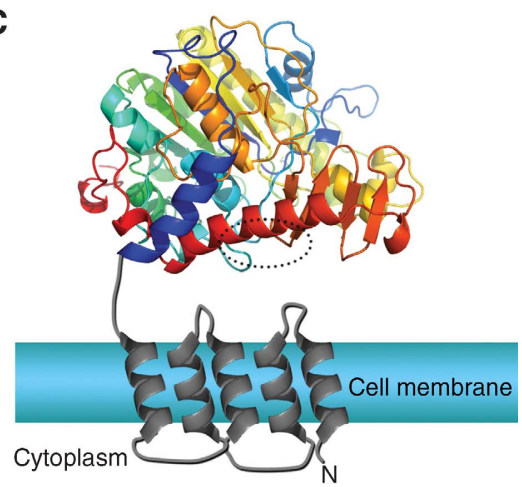

Figure 7 Crystal structure of $\mathrm{LtaS}_{215-649}$. (A) The protein backbone is rendered as a secondary structure cartoon colour ramped from blue at the $\mathrm{N}$ terminus to red at the $\mathrm{C}$ terminus. Orthogonal views are shown with the secondary structure elements highlighted in the middle panel. (B) The active site of LtaS shown in two views, with residues coordinating the magnesium ion in the active site drawn as sticks with the final REFMAC-weighted $F_{\text {obs }}-F_{\text {calc }}$ electron density map contoured at 1.5 sigma and shown as blue wireframe. (C) The domain arrangement of fulllength LtaS is illustrated schematically. The N-terminal, trans-membrane spanning helices are modelled, and coloured grey, in the context of a biological membrane. The structure of the C-terminal, catalytic domain of LtaS is drawn as a cartoon, colour-ramped in rainbow manner from $\mathrm{N}$ (blue) to $\mathrm{C}$ terminus (red). The location of the active site is highlighted by a dotted circle.

C $\alpha$-hydroxyformylglycine in sulphatases (Supplementary Figure 1B) and the nucleophilic serine in APs and given that a constellation of Thr297-surrounding amino acids (Glu253, Asp471 and His472) are also conserved in the AP superfamily, we propose that the reaction cycle of LtaS also proceeds through a covalent enzyme intermediate, the adduct of which is likely to be glycerol phosphate. The electron density in the crystallographic maps clearly shows that Thr297 is modified by phosphorylation, not glycerophosphorylation, and MALDI-TOF mass spectroscopy of the protein shows two peaks with a mass difference of $79 \mathrm{Da}$ (peaks at 49741 and $49819 \mathrm{kDa}$, respectively), consistent only with phosphorylation of Thr297. Adjacent to phosphoThr297 are three water molecules, the positions of two of which may mimic the hydroxyls of a covalently attached glycerol phosphate. One water is in hydrogen bond contact with the side chains of His343, Asn345 and Arg352, the second is in contact with the guanidinium group of Arg352 and the O3P of phospho-Thr297 and the third water contacts the carboxylate of Glu253 and the main chain carbonyl of Thr408. His343, Asn345, Arg352 and Trp350-which sits directly above the phosphoryl group-are highly conserved in LtaS homologues, suggesting that these amino acids have a more important function in catalysis than a simple hydration sphere. Trp350, whose $\mathrm{N} \varepsilon$ atom contacts two of the phosphoryl oxygens and thus presumably participates in poly(GroP) formation (Figure 7B), is replaced in AP by
Arg166, which makes contact with the phosphoryl group during all steps of the reaction cycle (Galperin and Jedrzejas, 2001).

The phosphorylation of Thr297 appears to mimic an intermediate state of the formation of poly(GroP). The source of the phosphorylation, however, is not known. The depth of the pocket in which Thr297 is situated at the base reduces the possibility that LtaS is phosphorylated by a kinase, but instead the source of phosphorylation is likely to be a small molecule phosphodonor, which can phosphorylate two-component response regulators (McCleary et al, 1993). The presence of a phosphoryl group on the active centre of the enzyme appears to inhibit LtaS effectively as we could not produce poly(GroP) from glycerol phosphate. LtaS thus seems to have evolved to retain some features of alkaline phosphatase-type enzymes (e.g., covalent phosphoryl enzyme intermediate) and others from the arylsulphatase family (e.g., one-metal hydrolysis, not two or three metal hydrolysis). In fact, the absolute biochemical reaction catalysed by LtaS is not known: most likely the $3^{\prime}$ hydroxyl of an incoming glycerol phosphate moiety attacks the threonylglycerophosphate adduct to effect the elimination reaction. Such an in-line attack also invokes a role in catalysis for Lys296, invariant in LtaS homologues, yet its side chain is directed away from the active site ion in our structure. The pocket that is lined by His343, Asn345 and Arg352 is of ideal volume for only a single glycerol (Supplementary Figure 2A, B), 
implying that the growing chain of poly(GroP) is the nucleophile in the second stage of the reaction. However, the precise role (indeed, its actual identity) of the bound metal ion in catalysis, how the growing chain is recognized by LtaS and the enzymatic mechanism of poly(GroP) formation and its elimination from LtaS remain unknown and are the subject of further biochemical experimentation.

\section{Conclusions}

Despite their importance as almost ubiquitous and abundant components of the walls of Gram-positive bacteria, the functions of TAs have remained the subject of speculation for several decades. Why organisms such as $B$. subtilis produce two distinct forms of TA, linked to the wall and the membrane, has also been unclear. We have now taken several major steps towards answering these questions. First, we have established that although both WTA and LTA are dispensable in $B$. subtilis, jointly, they are essential. This together with recent evidence that LTA is essential in S. aureus (which relies largely on the cell division apparatus to enlarge; Pinho and Errington, 2003; Gründling and Schneewind, 2007b) reinforces the notion that TA synthetic enzymes are good potential targets for antibiotics against Gram-positive pathogens. Elucidation of the crystal structure of the catalytic domain of LtaS and identification of its active site residue provide an important framework from which to launch drug discovery programmes. Second, we have shown that the major function of the LTA system lies in divalent cation homoeostasis, in accordance with earlier speculations (Fischer, 1988; Neuhaus and Baddiley, 2003). Third, we have discovered that the WTA and LTA systems have distinct roles in B. subtilis cell morphogenesis. It is well known that B. subtilis and many other rod-shaped bacteria have distinct morphogenetic systems involved in cell elongation and cell division (e.g., Begg et al, 1990; Daniel and Errington, 2003; Claessen et al, 2008). Previous work on the WTA system has pointed to a specialized role in elongation, manifested by both a rounded cell phenotype (Wagner and Stewart, 1991;

\section{References}

Archibald AR, Armstrong JJ, Baddiley J, Hay JB (1961) Teichoic acids and the structure of bacterial walls. Nature 191: 570-572

Arigoni F, Pogliano K, Webb CD, Stragier P, Losick R (1995) Localization of protein implicated in establishment of cell type to sites of asymmetric division. Science 270: 637-640

Barak I, Behari J, Olmedo G, Guzman P, Brown DP, Castro E, Walker D, Westpheling J, Youngman P (1996) Structure and function of the Bacillus SpoIIE protein and its localization to sites of sporulation septum assembly. Mol Microbiol 19: 1047-1060

Begg KJ, Takasuga A, Edwards DH, Dewar SJ, Spratt BG, Adachi H, Ohta T, Matsuzawa H, Donachie WD (1990) The balance between different peptidoglycan precursors determines whether Escherichia coli cells will elongate or divide. J Bacteriol 172: 6697-6703

Bhavsar AP, Brown ED (2006) Cell wall assembly in Bacillus subtilis: how spirals and spaces challenge paradigms. Mol Microbiol 60: 1077-1090

Bhavsar AP, Erdman LK, Schertzer JW, Brown ED (2004) Teichoic acid is an essential polymer in Bacillus subtilis that is functionally distinct from teichuronic acid. J Bacteriol 186: 7865-7873

Blackman SA, Smith TJ, Foster SJ (1998) The role of autolysins during vegetative growth of Bacillus subtilis 168. Microbiology 144: $73-82$
D’Elia et al, 2006a) and association of the synthetic enzyme complex with the elongation machinery (Formstone et al, 2008). We now show that the ltaS mutants are more affected in processes associated with cell division. As WTA and LTA have identical structures in $B$. subtilis, apart from their linkage to PG and membrane, respectively, we assume that it is the spatial distribution of the two polymers that determines their specialized functions. In principle, this means that LTA remains tethered close to the cell membrane surface, whereas the WTA should migrate out through the wall as it matures. As cell division involves ingrowth of the septal membranes, LTA may be especially important in ensuring the availability of $\mathrm{Mg}^{2+}$ to synthetic enzymes acting at or near the leading edge of the invaginating septum.

To conclude, this study opens up a number of important new areas of research on the role of LTA and TAs generally, in cell morphogenesis, the cell cycle, sporulation and adaptation to environmental conditions, as well as providing new opportunities for drug discovery.

\section{Materials and methods}

For more information on Materials and methods, see Supplementary data. Strains and plasmids are listed in Supplementary Table 1.

\section{Supplementary data}

Supplementary data are available at The EMBO Journal Online (http://www.embojournal.org).

\section{Acknowledgements}

This study was supported by a grant from the UK Biotechnology and Biological Sciences Research Council. We thank the Electron Microscopy Research Services at Newcastle University for their help and Ian Selmes for technical support. We also thank the group members, particularly Ling Juan Wu, Mark Leaver and JanWillem Veening for helpful discussions and for a critical reading of the paper. We are grateful for access to the Diamond Light Source and its beamline staff for help during data collection. Coordinates for the crystal structure of LtaS are deposited in the Protein Databank with the accession number 2 w8d.

Boltes I, Czapinska H, Kahnert A, von Bülow R, Dierks T, Schmidt B, von Figura K, Kertesz MA, Usón I (2001) 1.3 A structure of arylsulfatase from Pseudomonas aeruginosa establishes the catalytic mechanism of sulfate ester cleavage in the sulfatase family. Structure 9: 483-491

Bond CS, Clements PR, Ashby SJ, Collyer CA, Harrop SJ, Hopwood JJ, Guss MJ (1997) Structure of a human lysosomal sulphatase. Structure 5: 227-289

Carballido-López R (2006) The bacterial actin-like cytoskeleton. Microbiol Mol Biol Rev 70: 888-909

Carballido-López R, Formstone A, Li Y, Ehrlich SD, Noirot P, Errington J (2006) Actin homolog MreBH governs cell morphogenesis by localization of the cell wall hydrolase LytE. Dev Cell 11: 399-409

Carniol K, Ben-Yehuda S, King N, Losick R (2005) Genetic dissection of the sporulation protein SpoIIE and its role in asymmetric division in Bacillus subtilis. J Bacteriol 187: 3511-3520

Cheung HY, Freese E (1985) Monovalent cations enable cell wall turnover of the turnover-deficient lyt-15 mutant of Bacillus subtilis. J Bacteriol 161: 1222-1225

Claessen D, Emmins R, Hamoen LW, Daniel RA, Errington J, Edwards DH (2008) Control of the cell elongation-division cycle by shuttling of PBP1 protein in Bacillus subtilis. Mol Microbiol 68: 1029-1046 
Cowan JA (1995) Biological Chemistry of Magnesium. New York: Wiley-VCH

D’Elia MA, Millar KE, Beveridge TJ, Brown ED (2006a) Wall teichoic acid polymers are dispensable for cell viability in Bacillus subtilis. J Bacteriol 188: 8313-8316

D’Elia MA, Pereira MP, Chung YS, Zhao W, Chau A, Kenney TJ, Sulavik MC, Black TA, Brown ED (2006b) Lesions in teichoic acid biosynthesis in Staphylococcus aureus lead to a lethal gain of function in the otherwise dispensable pathway. $J$ Bacteriol 188: 4183-4189

Dajkovic A, Lutkenhaus J (2006) Z ring as executor of bacterial cell division. J Mol Microbiol Biotechnol 11: 140-151

Daniel RA, Errington J (2003) Control of cell morphogenesis in bacteria: two distinct ways to make a rod-shaped cell. Cell 113: 767-776

Den Blaauwen T, de Pedro MA, Nguyen-Disteche M, Ayala JA (2008) Morphogenesis of rod-shaped sacculi. FEMS Microbiol Rev 32: 321-344

Dziarski R (2003) Recognition of bacterial peptidoglycan by the innate immune system. Cell Mol Life Sci 60: 1793-1804

Errington J (1993) Bacillus subtilis sporulation: regulation of gene expression and control of morphogenesis. Microbiol Mol Biol Rev 57: $1-33$

Errington J (2003) Regulation of endospore formation in Bacillus subtilis. Nat Rev Microbiol 1: 117-126

Fedtke I, Götz F, Peschel A (2004) Bacterial evasion of innate host defenses-the Staphylococcus aureus lesson. Int J Med Microbiol 294: $189-194$

Fischer W (1988) Physiology of lipoteichoic acids in bacteria. Adv Microb Physiol 29: 233-302

Fittipaldi N, Sekizaki T, Takamatsu D, Harel J, Domínguez-Punaro Mde L, Von Aulock S, Draing C, Marois C, Kobisch M, Gottschalk M (2008) D-alanylation of lipoteichoic acid contributes to the virulence of Streptococcus suis. Infect Immun 76: 3587-3594

Formstone A, Carballido-Lopez R, Noirot P, Errington J, Scheffers D-J (2008) Localization and interactions of teichoic acid synthetic enzymes in Bacillus subtilis. J Bacteriol 190: 1812-1821

Formstone A, Errington J (2005) A magnesium-dependent mreB null mutant: implications for the role of mreB in Bacillus subtilis. Mol Microbiol 55: 1646-1657

Galperin MY, Jedrzejas MJ (2001) Conserved core structure and active site residues in alkaline phosphatase superfamily enzymes. Proteins 45: 318-324

Glenn AR, Mandelstam J (1971) Sporulation in Bacillus subtilis 168. Comparison of alkaline phosphatase from sporulating and vegetative cells. Biochem J 123: 129-138

Gross M, Cramton SE, Götz F, Peschel A (2001) Key role of teichoic acid net charge in Staphylococcus aureus colonization of artificial surfaces. Infect Immun 69: 3423-3426

Gründling A, Schneewind O (2007a) Genes required for glycolipid synthesis and lipoteichoic acid anchoring in Staphylococcus aureus. J Bacteriol 189: 2521-2530

Gründling A, Schneewind O (2007b) Synthesis of glycerol phosphate lipoteichoic acid in Staphylococcus aureus. Proc Natl Acad Sci USA 104: 8478-8483

Henriques AO, Glaser P, Piggot PJ, Moran Jr CP (1998) Control of cell shape and elongation by the rodA gene in Bacillus subtilis. Mol Microbiol 28: 235-247

Heptinstall S, Archibald AR, Baddiley J (1970) Teichoic acids and membrane function in bacteria. Nature 225: 519-521

Hilbert DW, Piggot PJ (2004) Compartmentalization of gene expression during Bacillus subtilis spore formation. Microbiol Mol Biol Rev 68: 234-262

Hirose I, Sano K, Shioda I, Kumano M, Nakamura K, Yamane K (2000) Proteome analysis of Bacillus subtilis extracellular proteins: a two-dimensional protein electrophoretic study. Microbiology 146: 65-75

Hofmann K, Stoffel W (1993) Tmbase-a database of membrane spanning proteins segments. Biol Chem Hoppe-Seyler 374: 166

Höltje JV (1998) Growth of the stress-bearing and shape-maintaining murein sacculus of Escherichia coli. Microbiol Mol Biol Rev 62: 181-203

Illing N, Errington J (1991) Genetic regulation of morphogenesis in Bacillus subtilis: roles of $\sigma^{\mathrm{E}}$ and $\sigma^{\mathrm{F}}$ in prespore engulfment. J Bacteriol 173: 3159-3169
Jervis AJ, Thackray PD, Houston CW, Horsburgh MJ, Moir A (2007) SigM-responsive genes of Bacillus subtilis and their promoters. J Bacteriol 189: 4534-4538

Jonas S, van Loo B, Hyvönen M, Hollfelder F (2008) A new member of the alkaline phosphatase superfamily with a formylglycine nucleophile: structural and kinetic characterisation of a phosphonate monoester hydrolase/phosphodiesterase from Rhizobium leguminosarum. J Mol Biol 384: 120-136

Jones LJ, Carballido-López R, Errington J (2001) Control of cell shape in bacteria: helical, actin-like filaments in Bacillus subtilis. Cell 104: 913-922

Khvorova A, Zhang L, Higgins ML, Piggot PJ (1998) The spoIIE locus is involved in the Spo0A-dependent switch in the location of FtsZ rings in Bacillus subtilis. J Bacteriol 180: $1256-1260$

Koch AL (1985) How bacteria grow and divide in spite of internal hydrostatic pressure. Can J Microbiol 31: 1071-1084

Koch AL (2003) Bacterial wall as target for attack: past, present, and future research. Clin Microbiol Rev 16: 673-687

Koch AL (2006) The exocytoskeleton. J Mol Microbiol Biotechnol 11: $115-125$

Kovacs M, Halfmann A, Fedtke I, Heintz M, Peschel A, Vollmer W, Hakenbeck R, Bruckner R (2006) A functional dlt operon, encoding proteins required for incorporation of $\mathrm{D}$-alanine in teichoic acids in Gram-positive bacteria, confers resistance to cationic antimicrobial peptides in Streptococcus pneumoniae. J Bacteriol 188: $5797-5805$

Kristian SA, Lauth X, Nizet V, Götz F, Neumeister B, Peschel A, Landmann R (2003) Alanylation of teichoic acids protects Staphylococcus aureus against Toll-like receptor 2-dependent host defense in a mouse tissue cage infection model. J Infect Dis 188: 414-423

Lazarevic V, Karamata D (1995) The tagGH operon of Bacillus subtilis 168 encodes a two-component $\mathrm{ABC}$ transporter involved in the metabolism of two wall teichoic acids. Mol Microbiol 16: 345-355

Leaver M, Errington J (2005) Roles for MreC and MreD proteins in helical growth of the cylindrical cell wall in Bacillus subtilis. Mol Microbiol 57: 1196-1209

Mauël C, Young M, Karamata D (1991) Genes concerned with synthesis of poly(glycerol phosphate), the essential teichoic acid in Bacillus subtilis strain 168, are organized in two divergent transcription units. J Gen Microbiol 137: 929-941

McCleary WR, Stock JB, Ninfa AJ (1993) Is acetyl phosphate a global signal in Escherichia coli. J Bacteriol 175: 2783-2798

Mendelson NH, Thwaites JJ, Favre D, Surana U, Briehl MM, Wolfe A (1985) Factors contributing to helical shape determination and maintenance in Bacillus subtilis macrofibres. Ann Inst Pasteur Microbiol 136A: 99-103

Millán JL (2006) Alkaline phosphatases: structure, substrate specificity and functional relatedness to other members of a large superfamily of enzymes. Purinergic Signal 2: 335-341

Morath S, Geyer A, Hartung T (2001) Structure-function relationship of cytokine induction by lipoteichoic acid from Staphylococcus aureus. J Exp Med 193: 393-397

Moszer I, Jones LM, Moreira S, Fabry C, Danchin A (2002) SubtiList: the reference database for the Bacillus subtilis genome. Nucleic Acids Res 30: 62-65

Neuhaus FC, Baddiley J (2003) A continuum of anionic charge: structures and functions of D-alanyl teichoic acids in Grampositive bacteria. Microbiol Mol Biol Rev 67: 686-723

Nouaille S, Commissaire J, Gratadoux JJ, Ravn P, Bolotin A, Gruss A, Le Loir Y, Langella P (2004) Influence of lipoteichoic acid D-alanylation on protein secretion in Lactococcus lactis as revealed by random mutagenesis. Appl Environ Microbiol 70: $1600-1607$

Oku Y, Kurokawa K, Matsuo M, Yamada S, Lee BL, Sekimizu K (2009) Pleiotropic roles of polyglycerolphosphate synthase of lipoteichoic acid in growth of Staphylococcus aureus cells. J Bacteriol 191: 141-151

Paulton RJ (1971) Nuclear and cell division in filamentous bacteria. Nat New Biol 231: 271-274

Perego M, Glaser P, Minutello A, Strauch MA, Leopold K, Fischer W (1995) Incorporation of D-alanine into lipoteichoic acid and wall teichoic acid in Bacillus subtilis. Identification of genes and regulation. J Biol Chem 270: 15598-15606 
Piggot PJ, Coote JG (1976) Genetic aspects of bacterial endospore formation. Microbiol Mol Biol Rev 40: 908-962

Pinho MG, Errington J (2003) Dispersed mode of Staphylococcus aureus cell wall synthesis in the absence of the division machinery. Mol Microbiol 50: $871-881$

Schaffer C, Messner P (2005) The structure of secondary cell wall polymers: how Gram-positive bacteria stick their cell walls together. Microbiology 151: 643-651

Schertzer JW, Brown ED (2003) Purified, recombinant TagF protein from Bacillus subtilis 168 catalyzes the polymerization of glycerol phosphate onto a membrane acceptor in vitro. J Biol Chem 278: 18002-18007

Schirner K, Errington J (2009) The cell wall regulator sI specifically suppresses the lethal phenotype of mbl mutants in Bacillus subtilis. J Bacteriol 191 (in press; doi:10.1128/JB.01497-08)

Seo HS, Michalek SM, Nahm MH (2008) Lipoteichoic acid is important in innate immune responses to Gram-positive bacteria. Infect Immun 76: 206-213

Smith TJ, Blackman SA, Foster SJ (2000) Autolysins of Bacillus subtilis: multiple enzymes with multiple functions. Microbiology 146: $249-262$

Stewart GC (2005) Taking shape: control of bacterial cell wall biosynthesis. Mol Microbiol 57: 1177-1181

Stragier P, Bouvier J, Bonamy C, Szulmajster J (1984) A developmental gene product of Bacillus subtilis homologous to the sigma factor of Escherichia coli. Nature 312: 376-378
Thompson LS, Beech PL, Real G, Henriques AO, Harry EJ (2006) Requirement for the cell division protein DivIB in polar cell division and engulfment during sporulation in Bacillus subtilis. J Bacteriol 188: 7677-7685

Vollmer W, Blanot D, de Pedro MA (2008) Peptidoglycan structure and architecture. FEMS Microbiol Rev 32: 149-167

Wagner PM, Stewart GC (1991) Role and expression of the Bacillus subtilis rodC operon. J Bacteriol 173: 4341-4346

Wecke J, Madela K, Fischer W (1997) The absence of D-alanine from lipoteichoic acid and wall teichoic acid alters surface charge, enhances autolysis and increases susceptibility to methicillin in Bacillus subtilis. Microbiology 143: 2953-2960

Wei Y, Havasy T, McPherson DC, Popham DL (2003) Rod shape determination by the Bacillus subtilis class $\mathrm{B}$ penicillin-binding proteins encoded by $p b p A$ and $p b p H$. J Bacteriol 185: 4717-4726

Weidenmaier C, Kokai-Kun JF, Kristian SA, Chanturiya T, Kalbacher H, Gross M, Nicholson G, Neumeister B, Mond JJ, Peschel A (2004) Role of teichoic acids in Staphylococcus aureus nasal colonization, a major risk factor in nosocomial infections. Nat Med 10: 243-245

Wu LJ, Errington J (1994) Bacillus subtilis SpoIIIE protein required for DNA segregation during asymmetric cell division. Science 264: $572-575$

Wu LJ, Errington J (1998) Use of asymmetric cell division and spoIIIE mutants to probe chromosome orientation and organization in Bacillus subtilis. Mol Microbiol 27: 777-786 